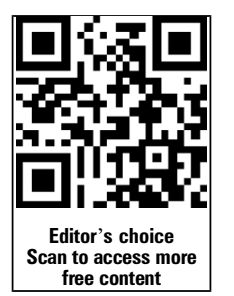

can to access more free content

- Additional material is published online only. To view please visit the journal online (http://dx.doi.org/10.1136/ bjsports-2014-094388).

${ }^{1}$ Department of Physical Therapy, University of British Columbia, Vancouver, British Columbia, Canada ${ }^{2}$ Department of Biology, University of British Columbia, Vancouver, British Columbia, Canada

${ }^{3}$ Department of Physical Therapy, University of Toronto, Toronto, Ontario, Canada

\section{Correspondence to} Yi-Wen Chen, Department of Physical Therapy, University of British Columbia, 2177 Wesbrook Mall Vancouver, British Columbia, Canada V6T 1Z3; yiwenchen@alumni.ubc. ca

Accepted 12 August 2015 Published Online First 17 September 2015
CrossMark

To cite: Chen Y-W, Hunt MA, Campbell KL, et al. Br J Sports Med 2016;50:397-407.

\title{
The effect of Tai Chi on four chronic conditions- cancer, osteoarthritis, heart failure and chronic obstructive pulmonary disease: a systematic review and meta-analyses
}

\author{
Yi-Wen Chen, ${ }^{1}$ Michael A Hunt, ${ }^{1}$ Kristin L Campbell, ${ }^{1}$ Kortni Peill, ${ }^{2}$ W Darlene Reid ${ }^{3}$
}

\begin{abstract}
Background Many middle-aged and older persons have more than one chronic condition. Thus, it is important to synthesise the effectiveness of interventions across several comorbidities. The aim of this systematic review was to summarise current evidence regarding the effectiveness of Tai Chi in individuals with four common chronic conditions-cancer, osteoarthritis (OA), heart failure (HF) and chronic obstructive pulmonary disease (COPD).
\end{abstract}

Methods 4 databases (MEDLINE, EMBASE, CINAHL and SPORTDiscus) were searched for original articles. Two reviewers independently screened the titles and abstracts and then conducted full-text reviews, quality assessment and finally data abstraction. 33 studies met the inclusion criteria. Meta-analyses were performed on disease-specific symptoms, physiological outcomes and physical performance of each chronic condition. Subgroup analyses on disease-specific symptoms were conducted by categorising studies into subsets based on the type of comparison groups.

Results Meta-analyses showed that Tai Chi improved or showed a tendency to improve physical performance outcomes, including 6-min walking distance (6MWD) and knee extensor strength, in most or all four chronic conditions. Tai Chi also improved disease-specific symptoms of pain and stiffness in OA.

Conclusions The results demonstrated a favourable effect or tendency of Tai Chi to improve physical performance and showed that this type of exercise could be performed by individuals with different chronic conditions, including COPD, HF and OA.

\section{INTRODUCTION}

Tai Chi, a physical activity which originated in ancient China, involves the interconnected concepts of Yin and Yang. ${ }^{1}$ The theory and principle of Tai Chi have been addressed in the literature, ${ }^{2-4}$ which emphasises the fusion of Yin and Yang into a single, ultimate and harmonious manner. Tai Chi is a popular exercise in Chinese society and has drawn increased attention in Western society, especially for older adults. All styles of Tai Chi consist of slow, gentle and flowing movements that involve strengthening, balance, postural alignment, mind concentration, relaxation and breath control. ${ }^{5}$ On the basis of these movement traits, Tai Chi is often classified as a low-to-moderate intensity physical activity and is a potentially suitable exercise for individuals in the general population, especially those who are middle-aged or older. ${ }^{67}$ Tai Chi has shown multifaceted benefits in improving health-related fitness, ${ }^{8}$ lower extremity muscle strength, ${ }^{9}{ }^{10}$ balance, ${ }^{11}$ fall prevention, ${ }^{12}$ cardiorespiratory function, ${ }^{13}{ }^{14}$ mental control $^{15}$ and flexibility. ${ }^{16}$

Tai Chi has been used as a complementary therapy in addition to more traditional Western healthcare approaches ${ }^{17}$ in several chronic conditions including cancer, ${ }^{18}{ }^{19}$ cardiovascular diseases $^{20-22}$ and arthritis. ${ }^{23} 24$ It has been shown that Tai Chi improves physiological and psychosocial outcomes in patients with chronic conditions. ${ }^{25}$ Given the fact that many middle-aged and older persons have more than one chronic condition, ${ }^{26}{ }^{27}$ it is important to synthesise the effectiveness of interventions across several comorbidities rather than reporting its effects solely within a single condition. The high prevalence of multimorbidity is demonstrated by a study of 980 Canadians reported that 9 of the 10 individuals had more than one chronic condition; 70\% of younger adults (aged 18-44 years) had more than one chronic condition and this prevalence rose to $98 \%$ in adults who were 65 years of age and older. ${ }^{28}$ Further, loss of function and physical limitations have been identified by patients as problem areas. Since Tai Chi can improve several attributes of fitness, it has potential as an exercise regimen for older individuals with multimorbidity.

We aimed to summarise current evidence regarding the effectiveness of Tai Chi in individuals with four common chronic conditionscancer, osteoarthritis (OA), heart failure (HF) and chronic obstructive pulmonary disease (COPD) some of these four chronic conditions may coexist. We included articles on conditions that had Tai Chi applied as an exercise intervention and met the inclusion criteria. Several conditions preliminarily searched for, that is, lung cancer, ischaemic heart disease, did not reveal any reports and thus were not considered in this systematic review. The evidence from this review will inform healthcare practitioners of important considerations when prescribing Tai Chi to people with multimorbidity, especially in older adults. This systematic review addressed the following questions: (1) Is Tai Chi an effective physical activity that improves symptoms, physical function, quality of life and depression in cancer, OA, HF and COPD? (2) Does Tai Chi have similar effects for the same outcome measures across different chronic conditions? 


\section{METHOD}

\section{Search strategy}

The following electronic databases were searched from their inception until 30 December 2014: MEDLINE, EMBASE, CINAHL and SPORTDiscus. Medical Subject Headings (MeSH) terms were employed to perform the search to retrieve citations and abstracts. "Tai Chi" was combined with each of the four chronic conditions: "cancer," "osteoarthritis," "heart failure" and "chronic obstructive pulmonary disease." The search strategy of each database is listed in online supplementary appendix 1. Additional searches were carried out by scanning the reference lists of related articles.

\section{Study criteria and selection}

Inclusion criteria for articles were studies that: (1) had participants with cancer or OA or HF or COPD; (2) used any form of Tai Chi, for example, modified Tai Chi or Qigong Tai Chi, as an intervention; (3) had a control group, other treatments or another exercise intervention as a comparison group; (4) reported outcomes of symptoms related to the respective chronic condition or outcomes of exercise training and (5) used a randomised controlled trial (RCT). Studies were excluded if they were not written in English.

Initially, two reviewers independently screened the titles and abstracts retrieved from the four databases. Next, two reviewers independently conducted full-text reviews and finally data abstractions. Disagreements were discussed between the two reviewers at each stage. A third reviewer was consulted to mediate decisions if consensus was not reached.

\section{Data extraction}

The following information was extracted in data abstraction forms: first author, year of publication, study purpose, participant characteristics, chronic conditions and diagnostic criteria, the details of Tai Chi (type, number of forms, duration, frequency, training length), details of comparison groups and outcomes (preintervention, postintervention and change scores, if available).

\section{Quality assessment}

The quality of included studies was assessed using the Physiotherapy Evidence-Based Database (PEDro) scale, ${ }^{29}$ which demonstrates good reliability. ${ }^{30}$ The PEDro scale consists of 11 criteria: eligibility criteria, random allocation, concealed allocation, baseline similarity, blinding of participants, blinding of therapists, blinding of assessors, follow-up, intention-to-treat analysis, between-group statistical comparisons, and point measures and measures of variability. One point is given for reporting of each criterion except item 1 (which refers to eligibility criteria) for a maximum score of 10 . Two reviewers independently assessed the study quality and disagreements were discussed until consensus was reached.

\section{Data management and statistical analysis}

Data were analysed using RevMan 5.2 (http://tech.cochrane.org/ revman/download). Meta-analyses were performed when at least two studies reported similar outcomes.

For meta-analyses, the mean changes (pre-post intervention) and the SDs of the mean changes of the outcome for the Tai Chi group and the comparison group were inputted into RevMan. Authors of 14 studies were approached to obtain data that were not available in the original paper, ${ }^{22} 31-47$ and six authors ${ }^{34-39}$ 45-47 provided data for the outcomes of interest.
When the SDs were not available after contacting the authors, SEs, CIs, $t$ values and $p$ values were used to calculate SDs. ${ }^{48}$

Meta-analyses were performed on disease-specific symptoms, physiological outcomes and the physical performance of each chronic condition. Subgroup analyses on disease-specific symptoms were conducted by categorising studies into subsets based on the type of comparison groups. For example, Tai Chi was compared with a control group in some studies, whereas in others the comparison group was another type of intervention. Thus, subgroup analyses were performed on each of these comparison groups. The overall effect for all studies, regardless of the comparison group, was calculated when no heterogeneity existed between subgroups.

Standardised mean difference (SMD) was used if the outcome was measured in a variety of ways. Mean difference (MD) was used if the outcome measurements were carried out using the same methodology. Inverse variance methods with a random effects model were used in the meta-analyses. The effect size of each study was presented as either SMD or MD with $95 \%$ CI. A p value of less than or equal to 0.05 was considered statistically significant for an overall effect. Heterogeneity among studies was identified using $\chi^{2}$ test and a $\mathrm{p}$ value of $<0.10$ was used to indicate significant heterogeneity among studies. Publication bias was assessed by visually examining a funnel plot. In addition, funnel plot asymmetry was statistically tested by Egger's test using Comprehensive Meta-Analysis (CMA) software V.2.2.064 (Biostat, Englewood, New Jersey, USA). A $\mathrm{p}$ value of $<0.05$ indicated the significance of publication bias.

\section{RESULTS}

\section{Study selection}

Searches of electronic databases produced 1102 citations (figure 1). Duplicates were excluded and reference lists yielded an additional five publications. Forty-one full-text articles were identified as potential inclusions after review of titles and abstracts. After full-text reviews, eight reports were excluded due to insufficient information or duplication of a conference proceeding (figure 1). Thirteen articles appeared to be similar in methodology but reported different outcome data in two or more publications. ${ }^{18} \quad 1921 \quad 23 \quad 34 \quad 35 \quad 41 \quad 46 \quad 47 \quad 49-52$ These were counted as individual studies in order to facilitate citation of outcome data. However, these reports were grouped in tables that described quality assessment (table 1) and participant characteristics (table 2). Of note, the search only revealed articles that reported the effect of Tai Chi for patients with breast cancer. Its use was not reported for any other types of cancer.

\section{Quality assessment}

The median PEDro score for included studies was 5 (IQR 56.75) (table 1). Owing to the nature of Tai Chi, blinding of respondents and therapists (PEDro items 5 and 6) is challenging and was not reported in all included studies. One study ${ }^{40}$ blinded assessors (PEDro item 7), whereas other studies utilised self-report outcomes, such that the participants were the assessors and hence nullified the possibility of a blinded assessor. Only one study ${ }^{32}$ scored 1 point on the PEDro scale and was excluded from quantitative syntheses due to missing data.

\section{Participants}

Sample sizes of included studies ranged from 11 to 206 (table 2). Breast cancer only included females (290 participants). Male-to-female ratios in the OA, HF and COPD studies were 81/452, 195/76 and 398/92, respectively. Mean ages of the 


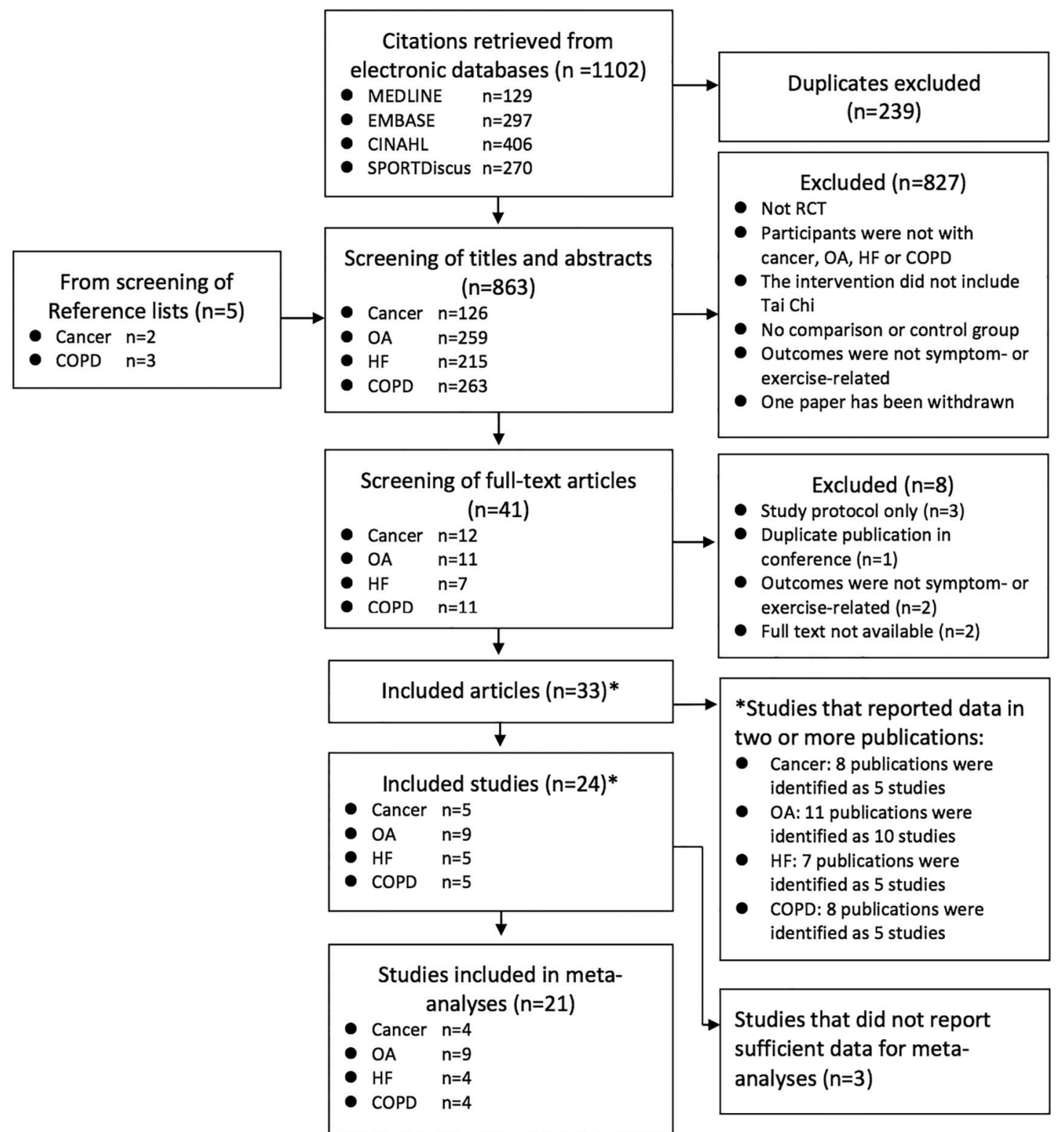

Figure 1 Flow chart showing different phases of the search strategy, screening and selection of studies (COPD, chronic obstructive pulmonary disease; HF, heart failure; $\mathrm{OA}$, osteoarthritis; $\mathrm{RCT}$, randomised controlled trial).

participants in the cancer, OA, HF and COPD studies were $53.9,68.8,68.4$ and 72.3 years, respectively. Diagnostic criteria of disease severity were reported in a variety of ways for each condition. For example, three of the five cancer studies ${ }^{32} 4153$ used cancer stage to denote cancer severity and recruited patients with cancer stages 0-IV. Two of the nine OA studies $^{24}{ }^{44}$ reported OA severity using the Kellgren-Lawrence grading scale and participants were rated as grade $2-4$, indicative of mild-to-severe OA. Other studies used symptoms as an indicator of OA severity, such as pain intensity on a visual analogue scale (VAS), and scored 4.4 of the 10 points $^{36}$ and 48.6 of the 100 points. ${ }^{54}$ Four of the five HF studies ${ }^{22} 435255$ used the New York Heart Association (NYHA) classification for HF and stated that the participants were in classes I-III, indicative of mild-to-moderate HF severity. Three of the five COPD studies ${ }^{40} 4256$ reported forced expiratory volume in $1 \mathrm{~s}\left(\mathrm{FEV}_{1}\right)$ indicative of participants with moderate-to-severe COPD and two studies reported stages of severity. ${ }^{35} 57$

\section{Characteristics of intervention}

Regarding the type of Tai Chi, the Yang style was used in 10 of the 24 studies ( 2 cancer $^{3241} ; 3 \mathrm{OA}^{31}{ }^{3644} ; 4 \mathrm{HF}^{43} 525558$ and $1 \mathrm{COPD}^{42}$ ). Sun style Tai Chi was used in four OA studies $^{23} 333738$ and two COPD studies. ${ }^{56}{ }^{57}$ Wu style Tai Chi was taught in only two studies $\left(1 \mathrm{OA}^{54}\right.$ and $\left.1 \mathrm{HF}^{22}\right)$. The type of Tai Chi was not stated in six studies. ${ }^{24} 3439404553$ The Tai Chi training programme ranged from 6 to 24 weeks and most were of 12-week duration. Session length ranged from 30 to 90 mins and most were of $1 \mathrm{~h}$ duration. Tai Chi training was usually offered 2-3 times per week. However, three studies 395354 provided Tai Chi once weekly and in one study, ${ }^{40}$ participants were asked to attend Tai Chi training every day.

Usual care or waitlist control was the most common comparison group, ${ }^{22-24} 3335-3739404252535657$ followed by comparison to another exercise intervention programme. ${ }^{31} 325558$ The effectiveness of Tai Chi was compared with that of educational programmes in four studies. ${ }^{38}{ }^{43-45}$ One cancer study ${ }^{41}$ used 
Table 1 Quality assessment-PEDro score

\begin{tabular}{|c|c|c|c|c|c|c|c|c|c|c|c|c|c|}
\hline \multirow[b]{2}{*}{ Condition } & \multirow[b]{2}{*}{ First author (year) } & \multicolumn{11}{|c|}{ Pedro score } & \multirow{2}{*}{$\begin{array}{l}\text { Total score } \\
\text { (items 2-11) }\end{array}$} \\
\hline & & 1 & 2 & 3 & 4 & 5 & 6 & 7 & 8 & 9 & 10 & 11 & \\
\hline \multirow[t]{5}{*}{ Cancer } & Galantino $(2003)^{32}$ & $\checkmark$ & $\checkmark$ & & & & & & & & & & 1 \\
\hline & $\begin{array}{l}\text { Mustian }\left(2004^{41} 2006^{18} 2008\right)^{19} \\
\text { Sprod }(2012)^{49}\end{array}$ & $\checkmark$ & $\checkmark$ & $\checkmark$ & $\checkmark$ & & & & & & $\checkmark$ & $\checkmark$ & 5 \\
\hline & Robins $(2013)^{39}$ & & $\checkmark$ & $\checkmark$ & $\checkmark$ & & & & & & $\checkmark$ & $\checkmark$ & 5 \\
\hline & Rausch $(2007)^{53}$ & $\checkmark$ & $\checkmark$ & & $\checkmark$ & & & & & $\checkmark$ & $\checkmark$ & $\checkmark$ & 5 \\
\hline & Campo $(2013)^{45}$ & $\checkmark$ & $\checkmark$ & $\checkmark$ & $\checkmark$ & & & & & & $\checkmark$ & $\checkmark$ & 5 \\
\hline \multirow[t]{9}{*}{$\mathrm{OA}$} & Adler $(2007)^{54}$ & $\checkmark$ & $\checkmark$ & $\checkmark$ & & & & & & $\checkmark$ & & $\checkmark$ & 4 \\
\hline & Brismée $(2007)^{36}$ & $\checkmark$ & $s$ & & $\checkmark$ & & & & & & $\checkmark$ & $\checkmark$ & 4 \\
\hline & Fransen $(2007)^{33}$ & $\checkmark$ & $\checkmark$ & $\checkmark$ & $\checkmark$ & & & & $\checkmark$ & $\checkmark$ & $\checkmark$ & $\checkmark$ & 7 \\
\hline & Hartman $(2000)^{31}$ & $\checkmark$ & $\checkmark$ & & $\checkmark$ & & & & $\checkmark$ & & $\checkmark$ & $\checkmark$ & 5 \\
\hline & Lee $(2009)^{24}$ & 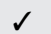 & $\checkmark$ & $\checkmark$ & 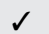 & & & & 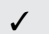 & $\checkmark$ & $\checkmark$ & $\checkmark$ & 7 \\
\hline & Song $\left(2003^{23} 2007\right)^{50}$ & $\checkmark$ & $\checkmark$ & $\checkmark$ & $\checkmark$ & & & & & & $\checkmark$ & $\checkmark$ & 5 \\
\hline & Song $(2010)^{38}$ & $\checkmark$ & $s$ & & $\checkmark$ & & & & & & $\checkmark$ & $\checkmark$ & 4 \\
\hline & Tsai $(2013)^{37}$ & & $\checkmark$ & & 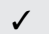 & & & & & $\checkmark$ & $\checkmark$ & $\checkmark$ & 5 \\
\hline & Wang $(2009)^{44}$ & $\checkmark$ & $\checkmark$ & $\checkmark$ & $\checkmark$ & & & & $\checkmark$ & $\checkmark$ & $\checkmark$ & $\checkmark$ & 7 \\
\hline \multirow[t]{5}{*}{$\mathrm{HF}$} & Barrow $(2007)^{22}$ & & $\checkmark$ & & $\checkmark$ & & & & & & $\checkmark$ & $\checkmark$ & 4 \\
\hline & Yeh $\left(2004^{52} 2008 a^{21} 2008 b\right)^{51}$ & $\checkmark$ & $\checkmark$ & $\checkmark$ & $\checkmark$ & & & & $\checkmark$ & $\checkmark$ & $\checkmark$ & $\checkmark$ & 7 \\
\hline & Yeh $(2011)^{43}$ & $\checkmark$ & $\checkmark$ & $\checkmark$ & $\checkmark$ & & & & $\checkmark$ & $\checkmark$ & $\checkmark$ & $\checkmark$ & 7 \\
\hline & Yeh $(2013)^{55}$ & $\checkmark$ & $\checkmark$ & $\checkmark$ & & & & & $\checkmark$ & $\checkmark$ & $\checkmark$ & $\checkmark$ & 6 \\
\hline & Caminiti $(2011)^{58}$ & $\checkmark$ & $\checkmark$ & & $\checkmark$ & & & & $\checkmark$ & & $\checkmark$ & $\checkmark$ & 5 \\
\hline \multirow[t]{5}{*}{ COPD } & Chan $\left(2010^{35} 2011^{34} 2013 a^{46} 2013 b\right)^{47}$ & $\checkmark$ & $\checkmark$ & $\checkmark$ & $\checkmark$ & & & & & $\checkmark$ & $\checkmark$ & $\checkmark$ & 6 \\
\hline & Leung $(2013)^{56}$ & $\checkmark$ & $\checkmark$ & $\checkmark$ & & & & & $\checkmark$ & $\checkmark$ & $\checkmark$ & $\checkmark$ & 6 \\
\hline & Yeh $(2010)^{42}$ & $\checkmark$ & $\checkmark$ & $\checkmark$ & & & & & & $\checkmark$ & $\checkmark$ & $\checkmark$ & 5 \\
\hline & Niu $(2014)^{40}$ & $\checkmark$ & $\checkmark$ & $\checkmark$ & $\checkmark$ & & & $\checkmark$ & $\checkmark$ & & $\checkmark$ & $\checkmark$ & 7 \\
\hline & $\mathrm{Ng}(2014)^{57}$ & $\checkmark$ & $\checkmark$ & $\checkmark$ & & & & & & $\checkmark$ & $\checkmark$ & $\checkmark$ & 5 \\
\hline \multicolumn{2}{|c|}{ Subtotals of each item } & 21 & 24 & 16 & 18 & 0 & 0 & 1 & 10 & 13 & 22 & 23 & Median 5 \\
\hline
\end{tabular}

psychosocial therapy as the comparison and one OA study ${ }^{54}$ used a non-physical recreational activity as the comparison group.

\section{Outcomes}

\section{Walk test}

Six-minute walking distance (6MWD) test was performed in cancer, OA, HF and COPD studies (figure 2). Analyses by each chronic condition demonstrated a favourable effect of Tai Chi on $6 \mathrm{MWD}$ in $\mathrm{HF} \quad(\mathrm{SMD}=1.58 ; 95 \%$ CI 0.70 to 2.45 ; $\mathrm{p}=0.0004)$ and COPD $(\mathrm{SMD}=0.37 ; 95 \%$ CI 0.01 to 0.73 ; $\mathrm{p}=0.05)$ studies, and trends towards improvement in one cancer report $(\mathrm{p}=0.09)$ and one OA $(\mathrm{p}=0.11)$ report.

\section{Muscle strength}

Knee extensor strength was assessed in OA, HF and COPD studies (figure 3). The subgroup (each chronic condition) difference was significant $(p=0.0009)$; Tai Chi improved strength even more so than the comparison group in one HF study $(\mathrm{SMD}=1.14 ; 95 \% \mathrm{CI} 0.60$ to $1.69 ; \mathrm{p}<0.0001)$ and one COPD study $(\mathrm{SMD}=1.64 ; 95 \% \mathrm{CI} 0.90$ to 2.39 ; $\mathrm{p}<0.0001)$, but it only showed a trend towards improvement in the OA studies $(\mathrm{p}=0.13)$ (figure 3).

\section{Timed Get Up and Go}

The aggregated data of the OA studies showed a favourable effect of Tai Chi versus the comparison groups on the Timed Get Up and Go (TUG) test $(\mathrm{MD}=0.56$; $95 \%$ CI 0.09 to 1.03 ; $\mathrm{p}=0.02)$. However, the HF study did not provide comparable evidence $(p=0.78)$ (figure 4$)$.
Quality of life

Meta-analyses performed on each chronic condition showed a significant effect of Tai Chi in OA studies $(\mathrm{SMD}=0.38 ; 95 \%$ CI 0.01 to $0.75 ; \mathrm{p}=0.05)$, and an improving trend for COPD and HF studies $(p=0.08 ; p=0.18$, respectively), whereas the cancer $(p=0.62)$ study did not provide evidence of Tai Chi improving quality of life more so than the comparison groups (figure 5).

\section{Depression}

Meta-analyses examining each of the chronic conditions only demonstrated a favourable effect of Tai Chi improving depression more so than the comparison groups in the HF studies $(\mathrm{SMD}=0.56 ; 95 \% \mathrm{CI} 0.07$ to $1.05 ; \mathrm{p}=0.03)$. Tai Chi showed a non-significant trend in COPD $(p=0.09)$ and OA $(p=0.32)$ studies. Of note, the two cancer studies demonstrated an effect in favour of the other treatment, which included stress management, on depression ( $\mathrm{SMD}=-0.97 ; 95 \% \mathrm{CI}-1.90$ to -0.05 ; $\mathrm{p}=0.04$ ) (figure 6).

\section{Symptoms of chronic conditions}

Two meta-analyses examined two major symptoms of OA, pain and stiffness. The meta-analyses, regardless of the comparison groups, showed evidence of Tai Chi improving pain $(\mathrm{SMD}=0.53 ; 95 \% \mathrm{CI} 0.32$ to $0.75 ; \mathrm{p}<0.0001$ ) (figure $7 \mathrm{~A})$ and stiffness $(\mathrm{SMD}=0.59 ; 95 \% \mathrm{CI} 0.31$ to $0.87 ; \mathrm{p}<0.0001$ ) (figure $7 \mathrm{~B})$. Subgroup analyses were performed that evaluated the effectiveness of Tai Chi compared with another intervention or compared with no intervention. Positive effects in favour of Tai Chi on pain were shown with either comparison group: other interventions $(\mathrm{SMD}=0.55 ; 95 \% \mathrm{CI} 0.09$ to $1.01 ; \mathrm{p}=0.02)$ or 
Table 2 Characteristics of participants

\begin{tabular}{|c|c|c|c|c|c|c|}
\hline $\begin{array}{l}\text { First author } \\
\text { (year) }\end{array}$ & Condition & $\begin{array}{l}\text { Sample size } \\
\mathrm{n}(\mathrm{M} / \mathrm{F})\end{array}$ & $\begin{array}{l}\text { Dropout } \\
\mathrm{n}(\%)\end{array}$ & Mean age (SD) & $\begin{array}{l}\text { Disease severity } \\
\text { Mean (SD) }\end{array}$ & Comparison group(s) \\
\hline Galantino $(2003)^{32}$ & Breast cancer & $11(0 / 11)$ & $3(27.3)$ & Unknown & Stage II-IV & Walking \\
\hline $\begin{array}{l}\text { Mustian }\left(2004^{41} 2006^{18}\right. \\
2008)^{19} \text { Sprod }(2012)^{49}\end{array}$ & Breast cancer & $31(0 / 31)$ & $10(32.3)$ & $52(9)$ & Stage 0 -IIlb breast cancer & $\begin{array}{l}\text { Psychosocial support } \\
\text { therapy typical care Con }\end{array}$ \\
\hline Robins $(2013)^{39}$ & Breast cancer & $145(0 / 145)$ & $36(24.8)$ & 50 & Unknown & $\begin{array}{l}\text { Spiritual growth group/ } \\
\text { Con }\end{array}$ \\
\hline Rausch $(2007)^{53}$ & Breast cancer & $40(0 / 40)$ & $11(27.5)$ & 49 & Stage | or || & $\begin{array}{l}\text { Spiritual growth group/ } \\
\text { Con }\end{array}$ \\
\hline Campo $(2013)^{45}$ & $\begin{array}{l}\text { Cancer } \\
\text { survivors }\end{array}$ & $63(0 / 63)$ & $9(14.3)$ & $67(7.15)$ & Unknown & Education Con \\
\hline Adler $(2007)^{54}$ & $\begin{array}{l}\text { Hip or knee } \\
\text { OA }\end{array}$ & $22(1 / 13)$ & $8(36.3)$ & $\begin{array}{l}\text { TC } 70.8(8.0) \\
\text { Con } 72.8(5.4)\end{array}$ & Pain intensity (0-100): $48.6(20.3)$ & $\begin{array}{l}\text { Non-physical recreationa } \\
\text { activity }\end{array}$ \\
\hline Brismée $(2007)^{36}$ & Knee OA & $41(7 / 34)$ & $10(24.3)$ & $\begin{array}{l}\text { TC } 70.8(9.8) \\
\text { Con } 68.8(8.9)\end{array}$ & $\begin{array}{l}\text { Pain VAS: TC } 4.7(2.6) \text {; Con } 4.2(1.8) \\
\text { WOMAC total }(26-130): \text { TC } 64.6(17.4) ; \text { Con } \\
59.6(15.2)\end{array}$ & Attention Con \\
\hline Fransen $(2007)^{33}$ & $\begin{array}{l}\text { Hip or knee } \\
\mathrm{OA}\end{array}$ & $152(40 / 112)$ & $11(7.2)$ & $\begin{array}{l}\text { TC } 70.8(6.3) \\
\text { Hydrotherapy } \\
70.0(6.3) \\
\text { Con } 69.6(6.1)\end{array}$ & $\begin{array}{l}\text { WOMAC-pain (0-100): TC } 40.3(19.0) \text {; } \\
\text { hydrotherapy } 38.2(17.4) ; \text { Con } 44.4(17.0)\end{array}$ & $\begin{array}{l}\text { Hydrotherapy/Con } \\
\text { (waitlist) }\end{array}$ \\
\hline Hartman $(2000)^{31}$ & $\begin{array}{l}\text { L/E or lumbar } \\
\text { spine } O A\end{array}$ & $35(5 / 28)$ & $2(5.7)$ & $\begin{array}{l}\text { TC } 68.6(7.9) \\
\text { Con } 67.5(6.1)\end{array}$ & Unknown & $\begin{array}{l}\text { Usual PA and routine } \\
\text { care }\end{array}$ \\
\hline Lee $(2009)^{24}$ & Knee OA & $44(3 / 41)$ & $3(6.8)$ & $\begin{array}{l}\text { TC } 70.2(4.8) \\
\text { Con } 66.9(6.0)\end{array}$ & $\begin{array}{l}\text { Kellgren-Lawrence, n (\%): Gr 2: TC } 14(48.3 \%) ; \\
\text { Con } 8(53.3 \%) \\
\text { Gr 3: TC } 13(45 \%) \text {; Con } 7(47 \%) \\
\text { Gr 4: TC } 2(6.9 \%) ; \text { Con } 0(0 \%)\end{array}$ & Con \\
\hline Song $\left(2003^{23} 2007\right)^{50}$ & Knee OA & $72(0 / 72)$ & $29(40.2)$ & $\begin{array}{l}\text { TC } 64.8(6.0) \\
\text { Con } 62.5(5.6)\end{array}$ & $\begin{array}{l}\text { K-WOMAC-pain (0-20): TC } 6.91 \text { (4.1); Con } \\
\text { 8.90 (5.1)Physical function: TC } 37.59 \text { (10.6); Con } \\
37.95 \text { (12.6) }\end{array}$ & Con \\
\hline Song $(2010)^{38}$ & Knee OA & $82(0 / 82)$ & $17(20.7)$ & $\begin{array}{l}\text { TC } 63.03(7.27) \\
\text { Con } 61.20(7.96)\end{array}$ & Unknown & $\begin{array}{l}\text { Self-help education } \\
\text { programme }\end{array}$ \\
\hline Tsai $(2013)^{37}$ & Knee OA & $55(15 / 40)$ & $10(18.2)$ & $\begin{array}{l}\text { TC } 78.89(6.91) \\
\text { Con } 78.93(8.30)\end{array}$ & WOMAC-pain: TC 6.96 (3.26); Con 7.65 (3.21) & Attention Con \\
\hline Wang $(2009)^{44}$ & Knee OA & $40(10 / 30)$ & $0(0)$ & $\begin{array}{l}\text { TC } 63(8.1) \\
\text { Con } 68(7.0)\end{array}$ & $\begin{array}{l}\text { Kellgren-Lawrence, } \mathbf{n}(\%) \text { : Gr 2: TC } 4(20 \%) ; \\
\text { Con } 3(15 \%) \\
\text { Gr 3: TC } 7(35 \%) ; \text { Con } 3(15 \%) \\
\text { Gr 4: TC } 9(45 \%) \text {; Con } 14(70 \%)\end{array}$ & $\begin{array}{l}\text { The wellness education } \\
\text { and stretching } \\
\text { programme }\end{array}$ \\
\hline Barrow $(2007)^{22}$ & $\mathrm{HF}$ & $65(53 / 12)$ & $13(20)$ & $\begin{array}{l}\text { TC } 68.4 \\
\text { Con } 67.9\end{array}$ & $\begin{array}{l}\text { NYHA symptom class II-III, MLHF mean score: } \\
\text { TC 35.1; Con } 34.9\end{array}$ & Con \\
\hline $\begin{array}{l}\text { Yeh }\left(2004^{52} 2008 a^{21}\right. \\
2008 b)^{51}\end{array}$ & $\mathrm{HF}$ & $30(19 / 11)$ & $0(0)$ & $64(13)$ & $\begin{array}{l}\text { Left ventricular ejection fraction,\%: TC } 24(7) \text {; } \\
\text { Con } 22(8) \\
\text { NYHA score: TC } 2.2(1.0) \text {; Con } 2.2(0.6)\end{array}$ & Usual care \\
\hline Yeh $(2011)^{43}$ & $\mathrm{HF}$ & $100(64 / 36)$ & $4(4)$ & $67(11)$ & $\begin{array}{l}\text { Left ventricular ejection fraction,\%: TC } 28 \text { (8); } \\
\text { Con } 29 \text { (7) } \\
\text { NYHA class HF I/IIIIII, n (\%): TC } 10(20) / 31 \\
(62) / 9(18) ; \text { Con } 10(20) / 32(64) / 8(16)\end{array}$ & Education Con \\
\hline Yeh $(2013)^{55}$ & $\mathrm{HF}$ & $16(8 / 8)$ & $0(0)$ & $66(12)$ & $\begin{array}{l}\text { NYHA class I/II/III, } \% \text { : TC 12/50/38; Con 25/63/ } \\
12\end{array}$ & Aerobic Ex \\
\hline Caminiti $(2011)^{58}$ & $\mathrm{HF}$ & $60(51 / 9)$ & $0(0)$ & $73.8(6)$ & Ejection fraction,\%: TC 33.6 (9); Con 32.8 (12) & Endurance training \\
\hline $\begin{array}{l}\text { Chan }\left(2010^{35} 2011^{34}\right. \\
\left.2013 a^{46} 2013 b\right)^{47}\end{array}$ & COPD & $206(187 / 19)$ & $48(23)$ & $\begin{array}{l}\text { TC } 71.7(8.2) \\
\text { Ex } 73.6(7.5) \\
\text { Con } 73.6(7.4)\end{array}$ & Mild $=15.5 \%$; moderate $=41.7 \%$; severe $=42.7 \%$ & $\mathrm{Ex} / \mathrm{Con}$ \\
\hline Leung $(2013)^{56}$ & COPD & $42(27 / 15)$ & $4(9.5)$ & $73(8)$ & $\%$ pred $\mathrm{FEV}_{1}=59(16) ; \mathrm{FEV} / \mathrm{FVC} \%=47(13)$ & Usual medical care \\
\hline Yeh $(2010)^{42}$ & COPD & $10(6 / 4)$ & $1(10)$ & $\begin{array}{l}\text { TC } 65(6) \\
\text { Con } 66(6)\end{array}$ & $\%$ pred $\mathrm{FEV}_{1}=50(12) ; \mathrm{FEV} / \mathrm{FVC}=0.63(0.14)$ & Usual care \\
\hline $\mathrm{Niu}(2014)^{40}$ & COPD & $40(3 / 37)$ & $1(2.5)$ & $\begin{array}{l}\text { TC } 59.7(12.34) \\
\text { Con } 61.3(12.92)\end{array}$ & $\begin{array}{l}\text { FEV }_{1}(\% \text { pred): TC } 41.9(24.60) \\
\text { Con } 43.7(23.08)\end{array}$ & Routine medical care \\
\hline $\mathrm{Ng}(2014)^{57}$ & COPD & $192(175 / 17)$ & $54(28.1)$ & $\begin{array}{l}\text { TC } 74.16(6.46) \\
\text { Con } 74.13(6.81)\end{array}$ & $\begin{array}{l}\text { TC: } \text { mild }=20 \% ; \text { moderate }=32 \% \text {; severe }=34 \% \text {; } \\
\text { very severe }=14 \% \\
\text { Con: } \text { mild }=20 \% ; \text { moderate }=49 \% \text {; severe }=27 \% \text {; } \\
\text { very severe }=4 \%\end{array}$ & $\begin{array}{l}\text { Pulmonary rehabilitation } \\
\text { programme }\end{array}$ \\
\hline
\end{tabular}




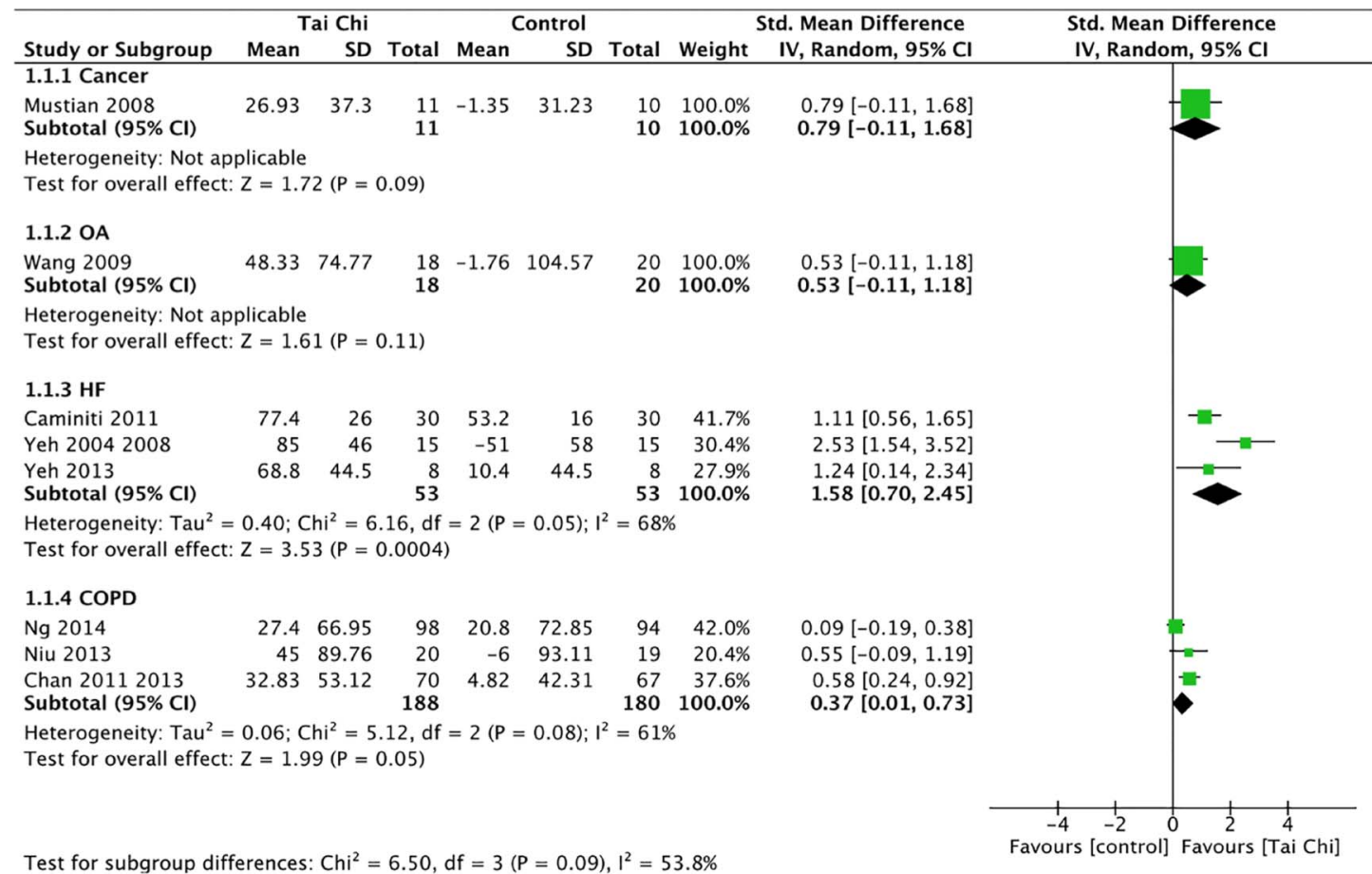

Figure 2 Forest plot for effects of Tai Chi on 6MWD (6MWD, 6-min walking distance; COPD, chronic obstructive pulmonary disease; HF, heart failure; OA, osteoarthritis).

no interventions $(\mathrm{SMD}=0.51 ; 95 \% \quad \mathrm{CI} \quad 0.24$ to 0.79 ; $\mathrm{p}=0.0003$ ) (figure 7A). Similarly, subgroup analyses showed a favourable effect of Tai Chi on stiffness regardless of the comparison group: other interventions $(\mathrm{SMD}=0.61 ; 95 \% \mathrm{CI} 0.25$ to $0.97 ; \mathrm{p}=0.0009)$ and no interventions ( $\mathrm{SMD}=0.57 ; 95 \% \mathrm{CI}$ 0.13 to $1.01 ; \mathrm{p}=0.01$ ) (figure $7 \mathrm{~B}$ ).

In COPD studies, meta-analysis showed a non-significant trend of Tai Chi alleviating dyspnoea versus the comparison groups $(\mathrm{MD}=0.51 ; 95 \% \mathrm{CI}-0.12$ to $1.15 ; \mathrm{p}=0.12$ ) (figure $7 \mathrm{C}$ ).

Meta-analyses could not be performed for cancer symptoms because no two studies reported similar outcomes. One study ${ }^{32}$ showed a trend of $75 \%$ participants experiencing less fatigue, measured by the Brief Fatigue Inventory (BFI), after either a 6-week Tai Chi or walking programme, but the small sample size $(n=8)$ most likely limited statistical power.

\section{Physiological outcomes}

The meta-analysis of two HF studies revealed no evidence that Tai Chi improved either systolic blood pressure (SBP) $(\mathrm{p}=0.80)$ (figure $8 \mathrm{~A})$ or diastolic blood pressure (DBP) $(p=0.33)$ (figure $8 \mathrm{~B})$. In addition, the meta-analysis of the other two HF studies reported a non-significant difference in peak oxygen uptake $\left(\mathrm{VO}_{2 \text { peak }}\right)$ from Tai Chi compared with either an exercise or a comparison group $(p=0.34)$ (figure 8C).

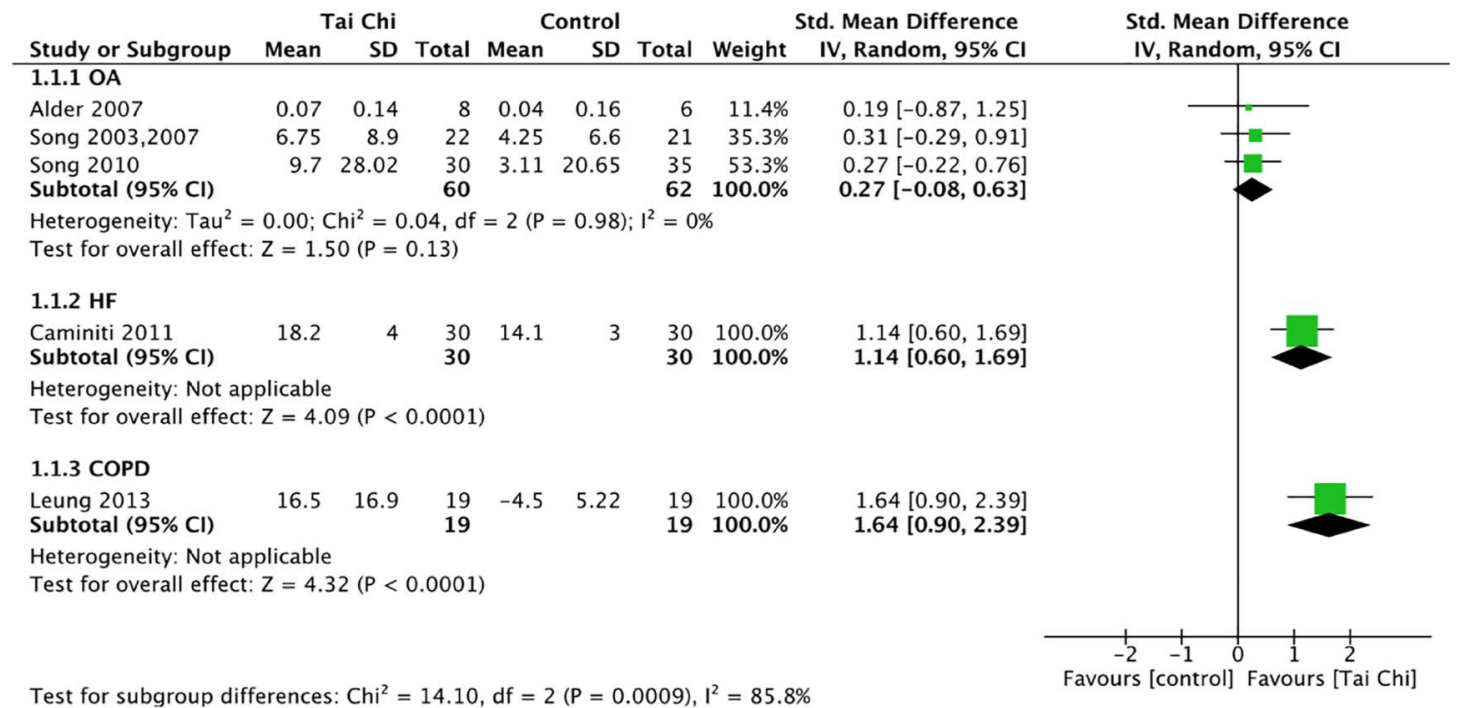

Figure 3 Forest plot for effects of Tai Chi on knee extensor strength (COPD, chronic obstructive pulmonary disease; HF, heart failure; OA, osteoarthritis). 


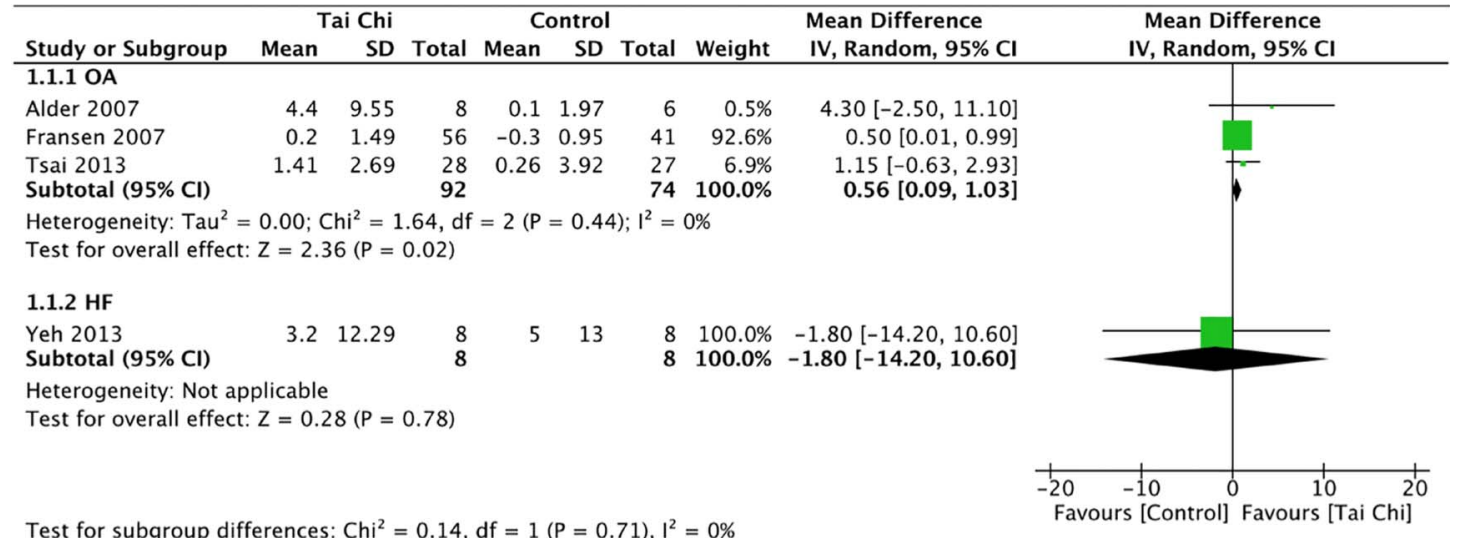

Figure 4 Forest plot for effects of Tai Chi on TUG test (HF, heart failure; OA, osteoarthritis; TUG, Timed Get Up and Go).

Meta-analysis of physical function in participants with OA assessed using the WOMAC showed a favourable effect of Tai Chi on improving physical function in participants with OA $(\mathrm{SMD}=0.70 ; 95 \%$ CI 0.47 to $0.93 ; \mathrm{p}<0.00001$ ) (figure $8 \mathrm{D})$. In addition, Tai Chi improved sit-to-stand times more so than the comparison groups $(\mathrm{SMD}=0.76 ; 95 \%$ CI 0.12 to 1.40 ; $\mathrm{p}=0.02$ ) (figure $8 \mathrm{E}$ ).

\section{Publication bias}

The funnel plot for quality of life did not show asymmetry (figure 9), which indicated no potential publication bias (Egger's test $\mathrm{p}=0.55)$. Funnel plots were not created in other outcomes, and therefore publication bias could not be determined due to the limited number of studies in each outcome measure. ${ }^{48}$

\section{DISCUSSION}

The most important findings of this systematic review and meta-analyses on 33 studies and 1584 participants were that Tai Chi showed favourable effects or modest trends on improving 6MWD, knee extensor strength and quality of life in most or all four chronic conditions: cancer, OA, HF and COPD. Our findings support the results of a previous systematic review that showed the effectiveness of Tai Chi on health outcomes in older patients with chronic conditions. ${ }^{25}$ These findings also complement previous systematic reviews that only examined the effect of Tai Chi in a single chronic condition. ${ }^{59-62}$ Moreover, Tai Chi provided greater improvements than comparison groups in other physical performance measures including decreased TUG times and shorter sit-to-stand times in participants with OA.

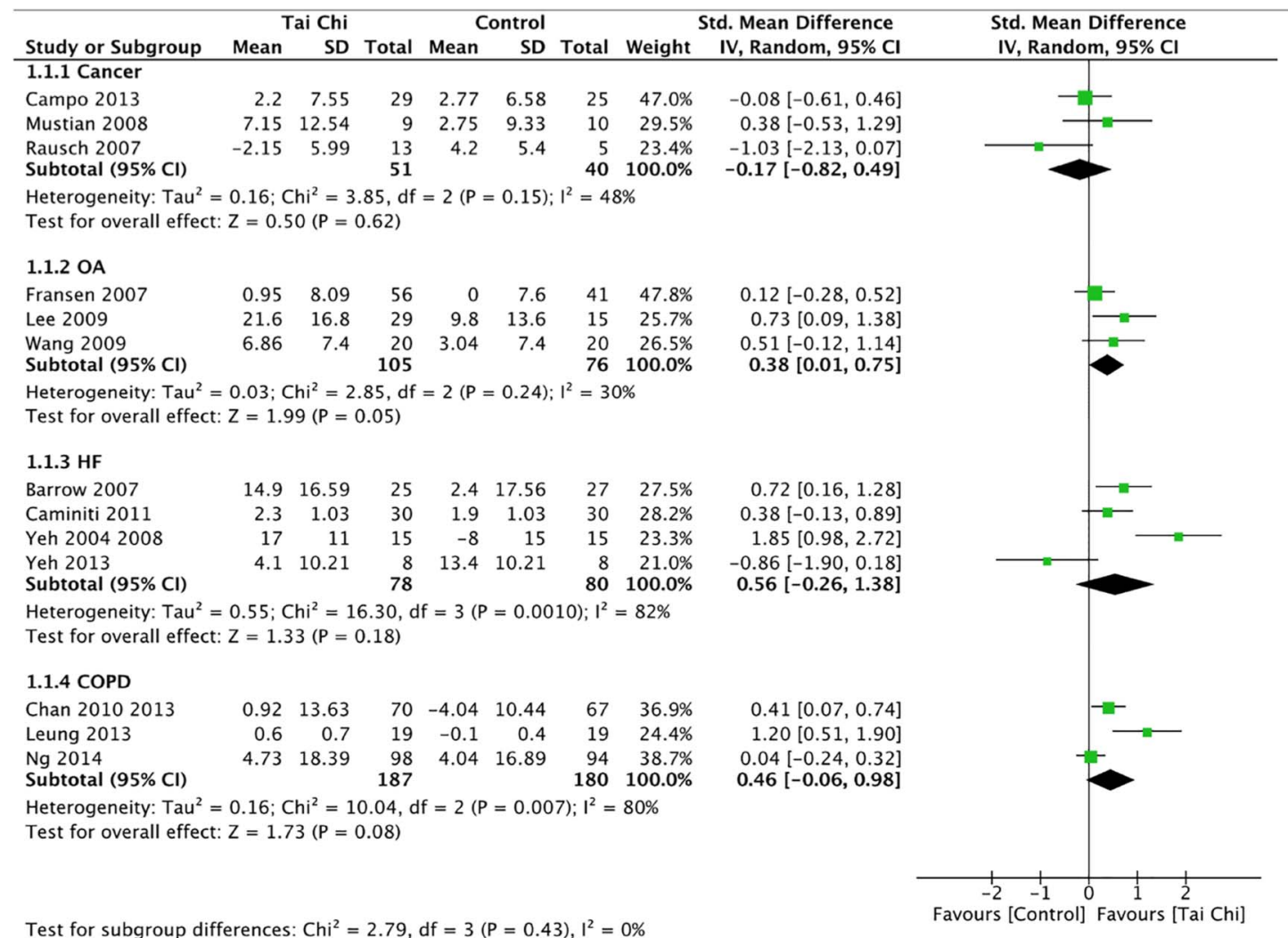

Figure 5 Forest plot for effects of Tai Chi on quality of life (COPD, chronic obstructive pulmonary disease; HF, heart failure; OA, osteoarthritis). 


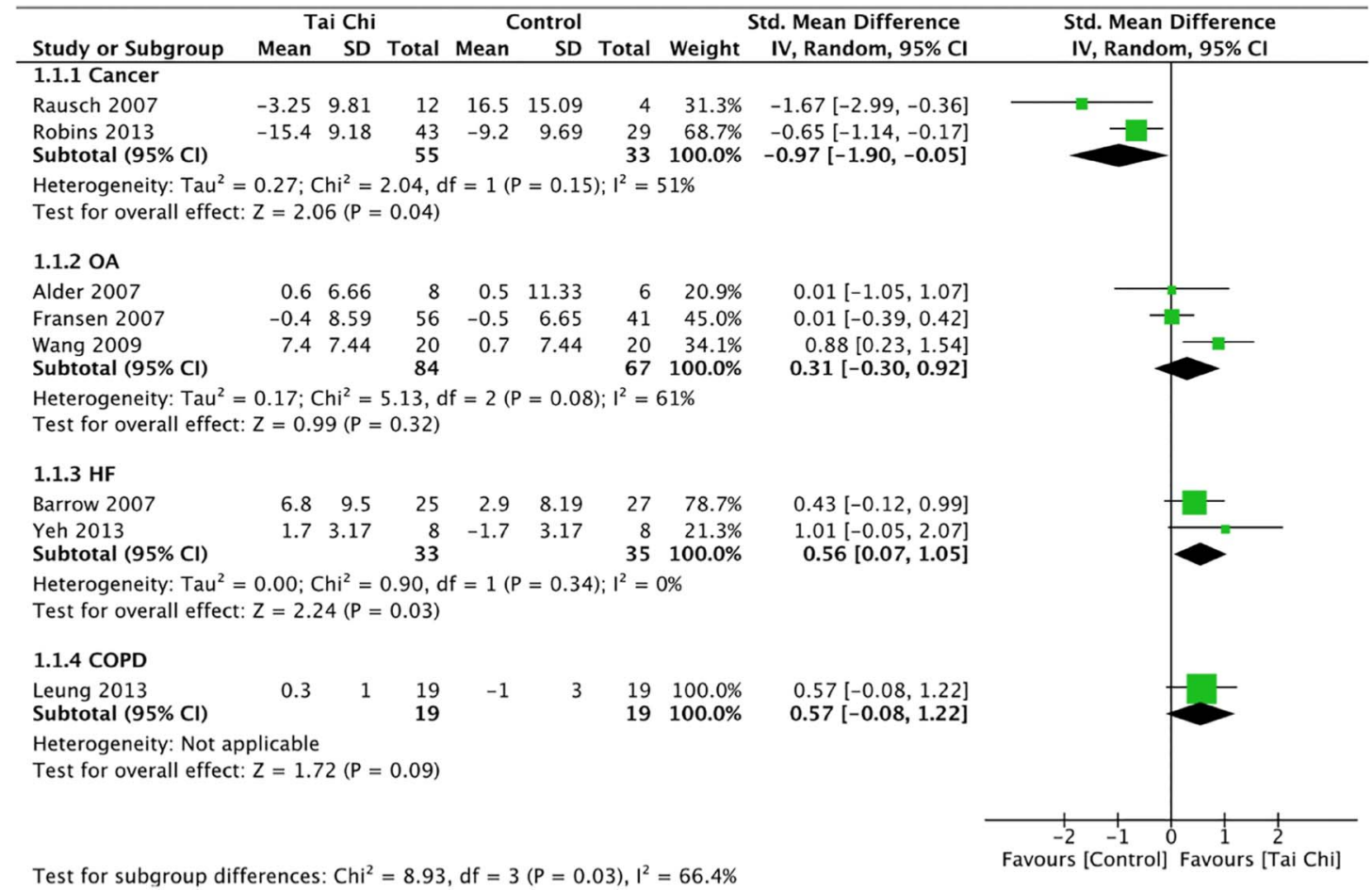

Finally, Tai Chi was more effective for improving pain and stiffness in OA compared with other interventions or controls.

Meta-analyses demonstrated that Tai Chi was more effective or showed modest trends compared with controls or other intervention groups towards improving the $6 \mathrm{MWD}$ in four chronic conditions. The $6 \mathrm{MWD}$ is a common primary outcome measure that is considered to evaluate functional exercise capacity. ${ }^{63}$ It shows moderate-to-high correlations with outcomes from peak cardiopulmonary exercise tests in participants with cardiac diseases $^{64}$ and COPD ${ }^{63}$ and has also been shown to increase after resistance training in participants with chronic $\mathrm{HF}^{65}$ The positive effect and trends of the 6MWD in all four conditions provide evidence that the exercise overload induced by Tai Chi was sufficient to improve functional exercise capacity in these chronic conditions.

Tai Chi improved the knee extensor muscle strength in participants with HF and COPD, but only showed a tendency towards improvement in participants with OA compared with other comparison groups and was not measured in participants with breast cancer. Although participants with knee OA have lower knee muscle strength, ${ }^{66}$ this impairment might be less than the weakness demonstrated in participants with $\mathrm{HF}^{67}$ and COPD, ${ }^{68}$ and thus a greater overload may be required than that provided by Tai Chi. Hence, Tai Chi appears to improve knee extensor strength in HF and COPD with more marginal benefits in OA. Further study is required to realise its impact on knee extensor strength in cancer survivors.

Other physical performance measures that require knee extensor strength improved more so after Tai Chi than other comparison groups in participants with OA. Most notably, TUG and sit-to-stand times showed greater improvements after Tai Chi than comparison groups in participants with OA. These findings may be attributed to the multifaceted training stimuli of Tai Chi that is directed towards improving balance and postural alignment in addition to strength. ${ }^{5}$ Although Tai Chi provides a minimal strength training stimulus, its positive influence on
TUG and sit-to-stand times provides strong evidence of its clinical impact on important functional measures in participants with $\mathrm{OA}$

The effect of Tai Chi on quality of life within a particular condition was less consistent. The most positive effects of Tai Chi on quality of life were shown in participants with OA. This is somewhat similar to a systematic review that examined participants with OA that showed improved physical quality of life, but not quality of life related to mental health. ${ }^{69}$ The differing results can be explained by the small sample sizes in the previous systematic review, which only included two studies and 84 participants versus 181 respondents in our systematic review. A strong trend was shown in participants with COPD, and the inclusion of RCTs that were written in other languages may have resulted in a significant overall effect towards improving quality of life in participants with COPD similar to another systematic review. ${ }^{60}$

In addition to the improvement in physical performance measures, symptoms either improved or did not differ after Tai Chi compared with the other treatment or a control group. Pain and stiffness in OA showed greater improvements after Tai Chi and dyspnoea showed a trend towards improvement in participants with COPD. Improved pain and stiffness in participants with OA may be attributed to increases in muscle strength, balance and/or postural alignment. ${ }^{70}$ From a clinical perspective, this evidence provides some assurance to the health professional that prescription of Tai Chi to persons living with OA and COPD has the potential to improve functional exercise capacity, but not at the expense of aggravating pain or causing undue dyspnoea.

\section{Limitations and strengths}

This meta-analysis has some limitations. First, only studies published in English were included due to the lack of two reviewers who were fluent in other languages. Second, with the exception of OA, there were limited numbers of RCTs in the other chronic conditions, especially regarding the availability of raw 
A. Pain

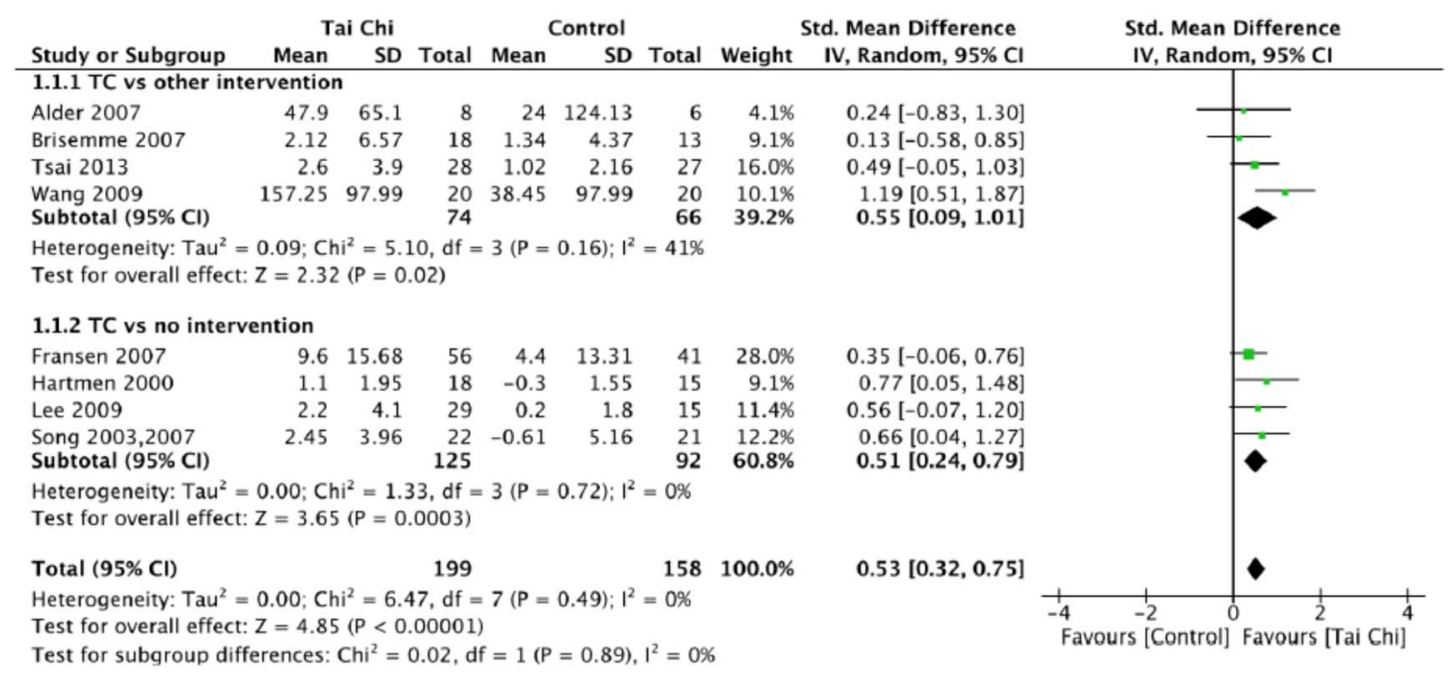

\section{B. Stiffness}

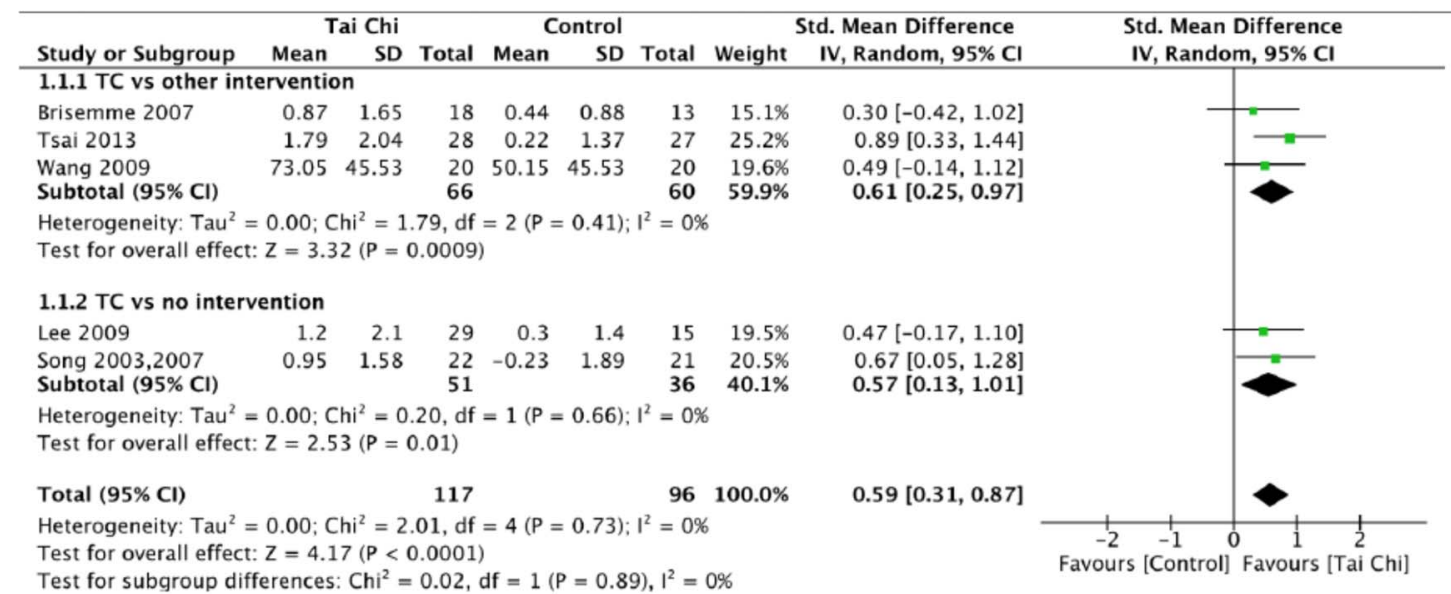

\section{Dyspnoea}

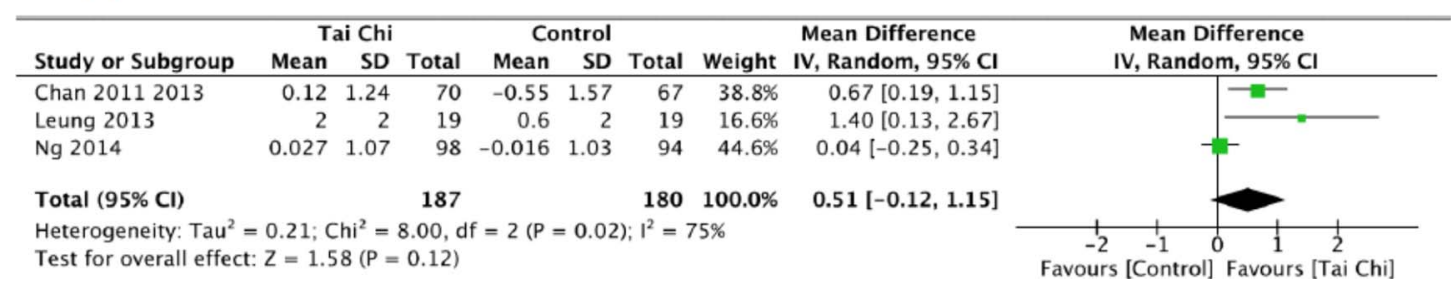

Figure 7 Forest plot for effects of Tai Chi on disease-specific symptoms: (A) pain; (B) stiffness; (C) dyspnoea.

data that could be included in the meta-analyses. Third, the sample sizes of included RCTs were quite small, contributing to limited power in most studies and in the aggregated data examined by meta-analyses. For example, the conflicting findings of the effect of Tai Chi on blood pressure in participants with HF are difficult to interpret because both reports had a small sample size. ${ }^{22} 58$ A fourth limitation is that participants in most studies represented patients who had one primary condition and may have excluded individuals with multimorbidity. Further study examining the effects of Tai Chi on multimorbidity is required to substantiate its benefits in this potentially complex patient group. Finally, the diversity of the type and parameters of Tai Chi in the included studies also limits the ability to make firm conclusions regarding the recommended Tai Chi exercise prescription for each chronic condition.

A key strength of this meta-analysis was the examination of the evidence regarding Tai Chi by reporting the symptoms, physiological and exercise outcomes of four common chronic conditions. Individuals with different chronic conditions may have different mechanisms that contribute to symptoms, impaired physical performance or quality of life. However, in people with multimorbidity, these underlying factors may also be inseparable or difficult to identify. Therefore, this systematic review offers a reasonable starting point to begin the quest for 
Figure 8 Forest plot for the effects of Tai Chi on physiological outcomes for heart failure (HF): (A) systolic blood pressure; (B) diastolic blood pressure; (C) peak oxygen uptake and osteoarthritis (OA): (D) WOMAC function; (E) sit to stand time.

\section{A. Systolic Blood Pressure (SBP) in HF}

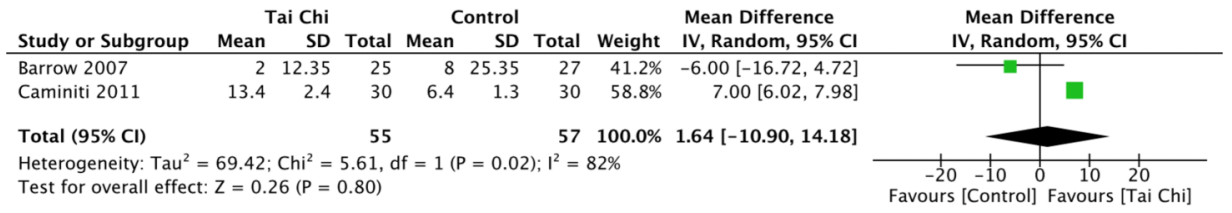

\section{B. Diastolic Blood Pressure (DBP) in HF}

\begin{tabular}{|c|c|c|c|c|c|c|c|c|c|}
\hline \multirow[b]{2}{*}{ Study or Subgroup } & \multicolumn{3}{|c|}{ Tai Chi } & \multicolumn{3}{|c|}{ Control } & \multirow[b]{2}{*}{ Weight } & \multirow{2}{*}{$\begin{array}{l}\text { Mean Difference } \\
\text { IV, Random, } 95 \% \mathrm{CI}\end{array}$} & \multirow{2}{*}{$\begin{array}{c}\text { Mean Difference } \\
\text { IV, Random, } 95 \% \mathrm{CI}\end{array}$} \\
\hline & Mean & SD & Total & Mean & SD & Total & & & \\
\hline Barrow 2007 & 4 & 10.2 & 25 & 7 & 18.01 & 27 & $6.5 \%$ & $-3.00[-10.88,4.88]$ & \\
\hline Caminiti 2011 & 5.6 & 1.4 & 30 & 4.3 & 0.7 & 30 & $93.5 \%$ & $1.30[0.74,1.86]$ & \\
\hline Total $(95 \% \mathrm{Cl})$ & & & 55 & & & 57 & $100.0 \%$ & $1.02[-1.05,3.10]$ & \\
\hline $\begin{array}{l}\text { Heterogeneity: } \mathrm{Tau}^{2} \\
\text { Test for overall effec }\end{array}$ & $\begin{array}{l}1.12 ; C \\
Z=0.9\end{array}$ & $\begin{array}{l}C h i^{2}= \\
96(P=\end{array}$ & $\begin{array}{l}1.14, \mathrm{~d} \\
=0.33)\end{array}$ & $f=1$ & $=0.2$ & $9) ; 1^{2}=$ & $12 \%$ & & $\begin{array}{ccccc}-10 & -5 & 1 & 1 & 1 \\
\text { Favours [Contorl] } & \text { Favours [Tai Chi] }\end{array}$ \\
\hline
\end{tabular}

\section{Peak Oxygen Uptake $\left(\mathrm{VO}_{2 \text { peak }}\right)$ in $\mathrm{HF}$}

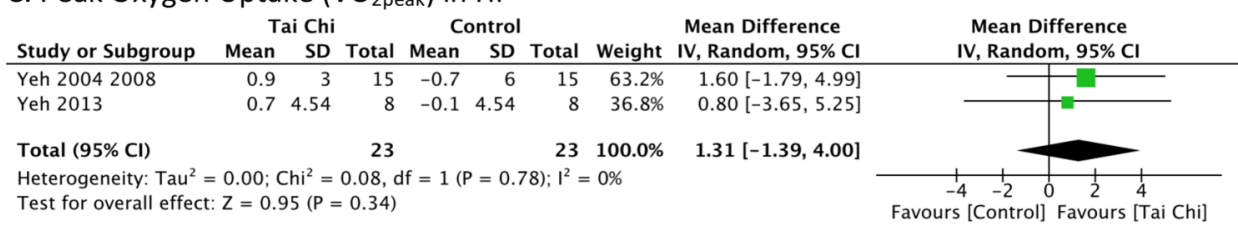

\section{WOMAC function in OA}

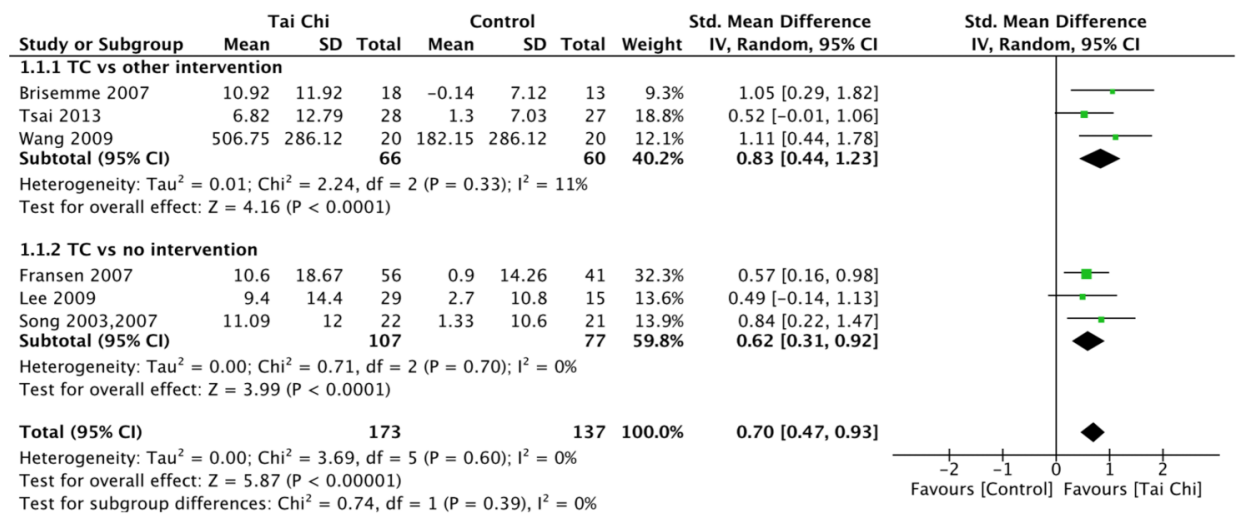

\section{E. Sit-to-stand time in OA}

\begin{tabular}{|c|c|c|c|c|c|c|c|c|c|c|}
\hline \multirow[b]{2}{*}{ Study or Subgroup } & \multicolumn{3}{|c|}{ Tai Chi } & \multicolumn{3}{|c|}{ Control } & \multicolumn{2}{|r|}{ Std. Mean Difference } & \multirow{2}{*}{\multicolumn{2}{|c|}{$\begin{array}{l}\text { Std. Mean Difference } \\
\text { IV, Random, } 95 \% \mathrm{CI}\end{array}$}} \\
\hline & Mean & SD & Total & Mean & SD & Total & Weight & IV, Random, 95\% Cl & & \\
\hline \multicolumn{11}{|c|}{ 1.1.1 TC vs other intervention } \\
\hline Tsai 2013 & 2.18 & 3.75 & 28 & 1.01 & 3 & 27 & $37.5 \%$ & $0.34[-0.19,0.87]$ & & $f=-$ \\
\hline Wang 2009 & 12.03 & 7.41 & 19 & 1.15 & 7.37 & 19 & $30.9 \%$ & $1.44[0.72,2.16]$ & & \\
\hline Subtotal $(95 \% \mathrm{Cl})$ & & & 47 & & & 46 & $68.4 \%$ & $0.86[-0.22,1.94]$ & & \\
\hline \multicolumn{11}{|c|}{$\begin{array}{l}\text { Heterogeneity: } \mathrm{Tau}^{2}=0.50 ; \mathrm{Chi}^{2}=5.80, \mathrm{df}=1(\mathrm{P}=0.02) ; \mathrm{I}^{2}=83 \% \\
\text { Test for overall effect: } \mathrm{Z}=1.57(\mathrm{P}=0.12)\end{array}$} \\
\hline \multicolumn{11}{|c|}{ 1.1.2 TC vs no intervention } \\
\hline $\begin{array}{l}\text { Hartmen } 2000 \\
\text { Subtotal }(\mathbf{9 5 \%} \mathrm{Cl})\end{array}$ & 0.8 & 1.85 & $\begin{array}{l}18 \\
18\end{array}$ & -0.5 & 2.46 & $\begin{array}{l}15 \\
15\end{array}$ & $\begin{array}{l}31.6 \% \\
31.6 \%\end{array}$ & $\begin{array}{l}0.59[-0.11,1.29] \\
0.59[-0.11,1.29]\end{array}$ & & \\
\hline \multicolumn{11}{|c|}{$\begin{array}{l}\text { Heterogeneity: Not applicable } \\
\text { Test for overall effect: } Z=1.65(P=0.10)\end{array}$} \\
\hline Total $(95 \% \mathrm{Cl})$ & & & 65 & & & 61 & $100.0 \%$ & $0.76[0.12,1.40]$ & & \\
\hline \multicolumn{9}{|c|}{$\begin{array}{l}\text { Heterogeneity: } \operatorname{Tau}^{2}=0.21 ; \mathrm{Chi}^{2}=5.90, \mathrm{df}=2(\mathrm{P}=0.05) ; \mathrm{I}^{2}=66 \% \\
\text { Test for overall effect: } \mathrm{Z}=2.31(\mathrm{P}=0.02) \\
\text { Test for subgroup differences: } \mathrm{Chi}^{2}=0.17, \mathrm{df}=1(\mathrm{P}=0.68), \mathrm{I}^{2}=0 \%\end{array}$} & \begin{tabular}{cc|}
1 & 1 \\
-4 & -2 \\
Favours [Control
\end{tabular} & $\begin{array}{lc}1 & 1 \\
0 & 2 \\
1] & \text { Favours [T }\end{array}$ \\
\hline
\end{tabular}

determining alternative exercise protocols, such as Tai Chi, for individuals who live with multimorbidity. Most of the included studies were moderate-to-high quality in terms of methodology (PEDro=4-7). Only one included study had poor quality ${ }^{32}$ and was excluded from quantitative syntheses due to missing data. This systematic review provided a quantitative synthesis of important physical performance outcomes, symptoms and quality of life. For some measures, evidence was limited due to the diverse outcome measurements among the conditions and small sample size. More rigorous RCTs with larger sample sizes are required to confirm the inconsistent findings among the included studies. Also, studies that examine patients with a broader range of diagnoses are required. For example, all of the studies examining cancer in this systematic review involved participants with breast cancer. It would be worthwhile to assess the effectiveness of Tai Chi on other types of cancer. 


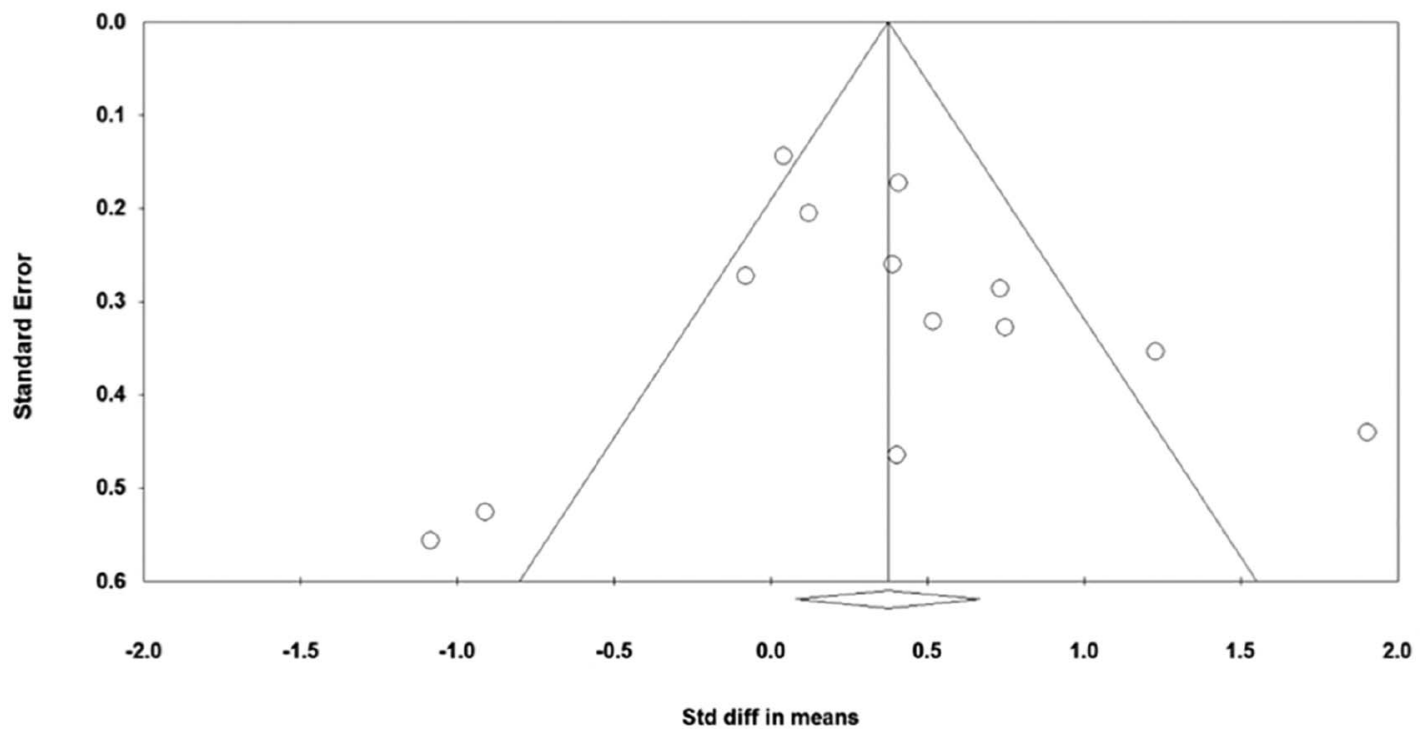

Figure 9 Funnel plot for quality of life.

In conclusion, the results provided evidence regarding a favourable effect or tendency of Tai Chi on improving the performance of $6 \mathrm{MWD}$, knee extensor strength and quality of life in people with cancer, OA, HF and COPD. Additionally, the meta-analyses showed the favourable effects of Tai Chi versus other interventions or no treatment on several disease-specific symptoms, including pain and stiffness. Taken together, Tai Chi demonstrates improvement in functional exercise capacity in individuals with different chronic conditions without aggravating symptoms of pain and dyspnoea. To summarise, Tai Chi appears to provide an adequate exercise stimulus and it could be a suitable exercise to prescribe for people with several comorbidities that include COPD, HF and OA.

\section{What are the findings?}

- Tai Chi can improve some physical performance outcomes in four chronic conditions (cancer, osteoarthritis, heart failure and chronic obstructive pulmonary disease) but not at the expense of worsening pain or dyspnoea.

- Tai Chi may provide a suitable exercise stimulus for people with several comorbidities.

\section{How might this impact on clinical practice in the future?}

- It is possible to consider prescribing Tai Chi in people with multimorbidity.

- Tai Chi is an exercise that is suitable to improve physical performance in middle-aged and older adults.

- Tai Chi can be a complementary therapy in some chronic conditions.

Acknowledgements The authors would like to thank the following people for providing data required to perform the meta-analyses: Sang-Cheol Bae, Jean-Michel Brismée, Aileen W K Chan, Jo Lynne W Robins, Chwan-Li Shen, Rhayun Song and Pao-Feng Tsai. They also acknowledge Alka Kurra and Carmen Ma for their assistance with data extraction and proofreading the manuscript.
Contributors Y-WC and WDR contributed to the conception and design of this systematic review. Y-WC conducted the search and analysed the data. Y-WC, MAH, KLC, KP and WDR extracted the data and completed the methodological quality assessment. Y-WC was primarily responsible for writing the manuscript. MAH, KLC, $\mathrm{KP}$ and WDR critically revised the manuscript.

Funding Y-WC is funded by the University of British Columbia and the BC Lung Association.

Competing interests None declared.

Provenance and peer review Not commissioned; externally peer reviewed.

\section{REFERENCES}

1 Horwood G. Tai Chi Chuan: the code of life revealing the deeper mysteries of China's ancient art for health. London, GBR: Jessica Kingsley Publishers, 2008.

2 Lan C, Lai JS, Chen SY. Tai Chi Chuan: an ancient wisdom on exercise and health promotion. Sports Med 2002;32:217-24.

3 Wolf SL, Coogler C, Xu T. Exploring the basis for Tai Chi Chuan as a therapeutic exercise approach. Arch Phys Med Rehabil 1997;78:886-92.

4 Koh TC. Tai Chi Chuan. Am J Chin Med 1981;9:15-22.

5 Irwin M, Pike J, Oxman M. Shingles immunity and health functioning in the elderly: Tai Chi Chih as a behavioral treatment. Evid Based Complement Alternat Med 2004;1:223-32.

6 Lan C, Chen SY, Lai JS. Relative exercise intensity of Tai Chi Chuan is similar in different ages and gender. Am J Clin Med 2004;32:151-60.

7 Fontana JA. The energy costs of a modified form of Tai Chi exercise. Nurs Res 2000;49:91-6.

8 Lan C, Lai JS, Wong MK, et al. Cardiorespiratory function, flexibility, and body composition among geriatric Tai Chi Chuan practitioners. Arch Phys Med Rehabil 1996;77:612-16.

9 Li JX, Xu DQ, Hong Y. Changes in muscle strength, endurance, and reaction of the lower extremities with Tai Chi intervention. J Biomech 2009;42:967-71.

10 Murphy L, Riley D, Rodgers J, et al. Effects of Tai Chi on balance, mobility, and strength among older persons participating in an osteoporosis prevention and education program. Explore (NY) 2005;1:192-3.

11 Wu G. Evaluation of the effectiveness of Tai Chi for improving balance and preventing falls in the older population-a review. J Am Geriatr Soc 2002;50:746-54.

12 Zhang JG, Ishikawa-Takata K, Yamazaki H, et al. The effects of Tai Chi Chuan on physiological function and fear of falling in the less robust elderly: an intervention study for preventing falls. Arch Gerontol Geriatr 2006;42:107-16.

13 Lan C, Lai JS, Chen SY, et al. 12-month Tai Chi training in the elderly: its effect on health fitness. Med Sci Sports Exerc 1998:30:345-51.

14 Audette JF, Jin YS, Newcomer R, et al. Tai Chi versus brisk walking in elderly women. Age Ageing 2006;35:388-93.

15 Li JX, Hong Y, Chan KM. Tai Chi: physiological characteristics and beneficial effects on health. Br J Sports Med 2001;35:148-56.

16 Hong Y, Li JX, Robinson PD. Balance control, flexibility, and cardiorespiratory fitness among older Tai Chi practitioners. Br J Sports Med 2000;34:29-34.

17 Barnes PM, Powell-Griner E, McFann K, et al. Complementary and alternative medicine use among adults: United States, 2002. Semin Integr Med 2004;2:54-71. 
18 Mustian KM, Katula JA, Zhao H. A pilot study to assess the influence of Tai Chi Chuan on functional capacity among breast cancer survivors. J Support Oncol 2006;4:139-45.

19 Mustian KM, Palesh OG, Flecksteiner SA. Tai Chi Chuan for breast cancer survivors. Med Sport Sci 2008;52:209-17.

20 Taylor-Piliae RE, Silva E, Sheremeta SP. Tai Chi as an adjunct physical activity for adults aged 45 years and older enrolled in phase III cardiac rehabilitation. Eur J Cardiovasc Nurs 2012;11:34-43.

21 Yeh G, Wayne P, Phillips R. Tai Chi exercise in patients with chronic heart failure. Med Sport Sci 2008;52:195-208.

22 Barrow DE, Bedford A, Ives G, et al. An evaluation of the effects of Tai Chi Chuan and Chi Kung training in patients with symptomatic heart failure: a randomised controlled pilot study. Postgrad Med J 2007;83:717-21.

23 Song R, Lee EO, Lam P, et al. Effects of Tai Chi exercise on pain, balance, muscle strength, and perceived difficulties in physical functioning in older women with osteoarthritis: a randomized clinical trial. J Rheumatol 2003;30:2039-44.

24 Lee HJ, Park HJ, Chae Y, et al. Tai Chi Qigong for the quality of life of patients with knee osteoarthritis: a pilot, randomized, waiting list controlled trial. Clin Rehabil 2009;23:504-11.

25 Wang C, Collet JP, Lau J. The effect of Tai Chi on health outcomes in patients with chronic conditions: a systematic review. Arch Intern Med 2004;164:493-501.

26 Grumbach K. Chronic illness, comorbidities, and the need for medical generalism. Ann Fam Med 2003;1:4-7.

27 Fillenbaum GG, Pieper CF, Cohen HJ, et al. Comorbidity of five chronic health conditions in elderly community residents: determinants and impact on mortality. J Gerontol A Biol Sci Med Sci 2000;55:M84-9.

28 Fortin M, Bravo G, Hudon C, et al. Prevalence of multimorbidity among adults seen in family practice. Ann Fam Med 2005;3:223-8.

29 PEDro scale. http://www.pedro.org.au/english/downloads/pedro-scale/ (accessed Oct 2013).

30 Maher CG, Sherrington C, Herbert RD, et al. Reliability of the PEDro scale for rating quality of randomized controlled trials. Phys Ther 2003;83:713-21.

31 Hartman CA, Manos TM, Winter C, et al. Effects of Tai Chi training on function and quality of life indicators in older adults with osteoarthritis. J Am Geriatr Soc 2000;48:1553-9.

32 Galantino ML, Capito L, Kane RJ, et al. The effects of Tai Chi and walking on fatigue and body mass index in women living with breast cancer: a pilot study. Rehabil Oncol 2003;21:17-22.

33 Fransen M, Nairn L, Winstanley J, et al. Physical activity for osteoarthritis management: a randomized controlled clinical trial evaluating hydrotherapy or Ta Chi classes. Arthritis Care Res 2007;57:407-14.

34 Chan AW, Lee A, Suen LK, et al. Tai Chi Qigong improves lung functions and activity tolerance in COPD clients: a single blind, randomized controlled trial. Complement Ther Med 2011;19:3-11.

35 Chan AW, Lee A, Suen LK, et al. Effectiveness of a Tai Chi Qigong program in promoting health-related quality of life and perceived social support in chronic obstructive pulmonary disease clients. Qual Life Res 2010;19:653-64.

36 Brismée JM, Paige RL, Chyu MC, et al. Group and home-based Tai Chi in elderly subjects with knee osteoarthritis: a randomized controlled trial. Clin Rehabil 2007;21:99-111.

37 Tsai PF, Chang JY, Beck C, et al. A pilot cluster-randomized trial of a 20-week Tai Chi program in elders with cognitive impairment and osteoarthritic knee: effects on pain and other health outcomes. J Pain Symptom Manage 2013;45:660-9.

38 Song $\mathrm{R}$, Roberts $\mathrm{BL}$, Lee EO, et al. A randomized study of the effects of Tai Chi on muscle strength, bone mineral density, and fear of falling in women with osteoarthritis. J Altern Complement Med 2010;16:227-33.

39 Robins JL, McCain NL, Elswick RK, et al. Psychoneuroimmunology-based stress management during adjuvant chemotherapy for early breast cancer. Evid Based Complement Alternat Med 2013;2013:372908. (accessed May 2014).

40 Niu R, He R, Luo BL, et al. The effect of Tai Chi on chronic obstructive pulmonary disease: a pilot randomised study of lung function, exercise capacity and diaphragm strength. Heart Lung Circ 2014;23:347-52.

41 Mustian KM, Katula JA, Gill DL, et al. Tai Chi Chuan, health-related quality of life and self-esteem: a randomized trial with breast cancer survivors. Support Care Cancer 2004;12:871-6.

42 Yeh GY, Roberts DH, Wayne PM, et al. Tai Chi exercise for patients with chronic obstructive pulmonary disease: a pilot study. Respir Care 2010;55:1475-82.

43 Yeh GY, McCarthy EP, Wayne PM, et al. Tai Chi exercise in patients with chronic heart failure: a randomized clinical trial. Arch Intern Med 2011;171:750-7.

44 Wang C, Schmid CH, Hibberd PL, et al. Tai Chi is effective in treating knee osteoarthritis: a randomized controlled trial. Arthritis Care Res 2009;61:1545-53.
45 Campo RA, O'Connor K, Light KC, et al. Feasibility and acceptability of a Tai Chi Chih randomized controlled trial in senior female cancer survivors. Integr Cancer Ther 2013;12:464-74.

46 Chan AW, Lee A, Lee DT, et al. Evaluation of the sustaining effects of Tai Chi Qigong in the sixth month in promoting psychosocial health in COPD patients: a single-blind, randomized controlled trial. ScientificWorldJournal 2013;2013:425082. (accessed May 2014).

47 Chan AW, Lee A, Lee DT, et al. The sustaining effects of Tai Chi Qigong on physiological health for COPD patients: a randomized controlled trial. Complement Ther Med 2013;21:585-94.

48 Higgins JP, Green S, eds. Cochrane handbook for systematic reviews of interventions Version 5.1.0 [updated March 2011]. The Cochrane Collaboration, 2011. http:// www.cochrane-handbook.org (accessed Jan 2014).

49 Sprod LK, Janelsins MC, Palesh OG, et al. Health-related quality of life and biomarkers in breast cancer survivors participating in Tai Chi Chuan. J Cancer Surviv 2012;6:146-54

50 Song $\mathrm{R}$, Lee EO, Lam $\mathrm{P}$, et al. Effects of a Sun-style Tai Chi exercise on arthritic symptoms, motivation and the performance of health behaviors in women with osteoarthritis. Taehan Kanho Hakhoe Chi 2007;37:249-56.

51 Yeh GY, Mietus JE, Peng CK, et al. Enhancement of sleep stability with Tai Chi exercise in chronic heart failure: preliminary findings using an ECG-based spectrogram method. Sleep Med 2008;9:527-36.

52 Yeh GY, Wood MJ, Lorell BH, et al. Effects of Tai Chi mind-body movement therapy on functional status and exercise capacity in patients with chronic heart failure: a randomized controlled trial. Am J Med 2004;117:541-8.

53 Rausch SM. Evaluating the psychosocial effects of two interventions, Tai Chi and spiritual growth groups in women with breast cancer [dissertation]. Richmond, VA: Virginia Commonwealth University, 2007.

54 Adler PA. The effects of Tai Chi on pain and function in older adults with osteoarthritis [dissertation]. Cleveland, OH: Case Western Reserve University, 2007.

55 Yeh GY, Wood MJ, Wayne PM, et al. Tai Chi in patients with heart failure with preserved ejection fraction. Congest Heart Fail 2013;19:77-84.

56 Leung RW, McKeough ZJ, Peters MJ, et al. Short-form Sun-style Tai Chi as an exercise training modality in people with COPD. Eur Respir J 2013;41:1051-7.

$57 \mathrm{Ng} \mathrm{L}$, Chiang LK, Tang R, et al. Effectiveness of incorporating Tai Chi in a pulmonary rehabilitation program for chronic obstructive pulmonary disease (COPD) in primary care-a pilot randomized controlled trial. Eur J Integr Med 2014;6:248-58

58 Caminiti G, Volterrani M, Marazzi G, et al. Tai Chi enhances the effects of endurance training in the rehabilitation of elderly patients with chronic heart failure. Rehabil Res Pract 2011;2011:761958. (accessed May 2014).

59 Pan L, Yan JH, Guo YZ, et al. Effects of Tai Chi training on exercise capacity and quality of life in patients with chronic heart failure: a meta-analysis. Euro J Heart Fail 2013;15:316-23.

60 Yan JH, Guo YZ, Yao HM, et al. Effects of Tai Chi in patients with chronic obstructive pulmonary disease: preliminary evidence. PLOS ONE 2013;8:e61806.

61 Zeng Y, Luo T, Xie H, et al. Health benefits of Qigong or Tai Chi for cancer patients: a systematic review and meta-analyses. Complement Ther Med 2014;22:173-86

62 Ye J, Cai S, Zhong W, et al. Effects of Tai Chi for patients with knee osteoarthritis: a systematic review. J Phys Ther Sci 2014;26:1133-7.

63 ATS Committee on Proficiency Standard for Clinical Pulmonary Function Laboratories. ATS statement: guidelines for the six-minute walk test. Am J Respir Crit Care Med 2002;166:111-17.

64 Bellet RN, Adams L, Morris NR. The 6-minute walk test in outpatient cardiac rehabilitation: validity, reliability and responsiveness-a systematic review. Physiotherapy 2012;98:277-86.

65 Hwang CL, Chien CL, Wu YT. Resistance training increases 6-minute walk distance in people with chronic heart failure: a systematic review. J Physiother 2010;56:87-96

66 Slemenda C, Brandt KD, Heilman DK, et al. Quadriceps weakness and osteoarthritis of the knee. Ann Intern Med 1997;127:97-104.

67 Pina I, Daoud S. Exercise and heart failure. Minerva Cardioangiol 2004;52: 537-46.

68 Hamilton AL, Killian KJ, Summers E, et al. Symptom intensity and subjective limitation to exercise in patients with cardiorespiratory disorders. Chest 1996;110:1255-63.

69 Lauche R, Langhorst J, Dobos $\mathrm{G}$, et al. A systematic review and meta-analysis of Tai Chi for osteoarthritis of the knee. Complement Ther Med 2013;21:396-406.

70 Bennell KL, Hinman RS. A review of the clinical evidence for exercise in osteoarthritis of the hip and knee. J Sci Med Sport 2011;14:4-9. 